\title{
Improvement Accuracy of Recognition Isolated Balinese Characters with Deep Convolution Neural Network
}

\author{
Ida Bagus Teguh Teja Murti*1 \\ Universitas Pendidikan Ganesha, Jalan Udayana No 11 Singaraja Bali 8116, (+62362) 22570 \\ E-mail : teguh.teja@gmail.com*1 \\ *Corresponding author
}

\begin{abstract}
The numbers of Balinese script and the low quality of palm leaf manuscripts provide a challenge for testing and evaluation for character recognition. The aim of high accuracy for character recognition of Balinese script,we implementation Deep Convolution Neural Network using SmallerVGG (Visual Geometry Group) Architectur for character recognition on palm leaf manuscripts. We evaluated the performance that methods and we get accuracy $87,23 \%$.
\end{abstract}

Keywords - Classfication, Deep Neural Network, Balinese Characters, Visual Geometry Group

\section{INTRODUCTION}

Isolated handwritten character recognition (IHCR) has been the subject of vigorous research in recent years. Some populer methods in this case are CNN for recognition numbers in the MNIST digits image database [1] . There are challenges in character recognition in some scripts, such as Chinese characters [2] and Balinese characters [3], which have more characters than numbers in MNIST. Beside numbers character there is issue in recognition character in document made palm leaf manuscript that is the condition of document has been degenerated. Therefore the researcher must carry out the process of digitizing the palm leaf manuscript, automatic analysis and the indexing system of the script simultaneously. The activity goal is bring added value to digital palm leaf manuscripts by developing tools for analyzing, developing and accessing quickly and efficiently script content. Making manuscript easier to obtain, read and understand by a wider audience or even scholars throughout the world.

Balinese manuscripts are written with knives made from iron (a special tool called Pengerupak) which is recorded on palm leaves. After writing the script is rubbed with natural coloring. Balinese palm leaves are written in Balinese script and in Balinese language. Writing in Balinese script, there is no space between words in one line of text. Some characters are written on the line above or below the base line of the text. Lontar written in ancient times literary texts are arranged in ancient Javanese Kawi and Sanskrit languages. Lontar contains important issues such as religion, holy characters, rituals, family genealogies, legal codes, medical agreements, art and architecture, calendars, prose, poetry, and even magic. Unfortunately, there are many Lontar, parts of collections in several museums or family owned, with damage due to age and due to inadequate storage.

Mostly Balinese have never read palm leaf manuscript because tradition constraints that consider it sacred and the language. They cannot access it contains important information and knowledge. Therefore, using the IHCR system can help to transcribe ancient documents and translate them into commonly used languages. The IHCR system is one of the most demanding systems to be developed in a collection of palm leaf manuscripts. Usually, the IHCR 
system uses extraction steps and feature classifications, but in [4] , the CNN method was used to directly process classification. This CNN method is implement on a Balinese script which has segmented each character or isolated character.

Pick out an efficient and powerful CNN architecture is very important to achieve high accuracy in IHCR and OCR. The CNN approach was chosen because it tends to be better on raw input pixels than features method. The purpose of developing an IHCR system torward Balinese script in palm leaf manuscripts, in this paper, apply on DCNN architecture is VGG [5] for character recognition of Balinese characters. We also compared the results with convolutional neural networks based on multiple layer perception (MLP) systems in Kesiman et al.

For IHCR Bali, there are 2 challenges: 1) poor quality of the text. Usually manuscript leaves are of poor quality because documents are degraded over time due to storage conditions. Manuscript palm leaves containing parts and artifacts that change color due to aging. They have low intensity, variation or contrast that is bad, random sounds, and fades [3]. Some deformations in character shapes are seen because of the combination and character fracture, various spaces between letters, and various spaces between lines (see Fig. 1). Balinese manuscripts on palm leaves offer real new challenges in the development of IHCR. 2 ) the complexity of Balinese script. Balinese script is considered one of the more complex scripts from Southeast Asia. The Balinese alphabet consists of $>100$ class characters including consonants, vowels, punctuation marks, and several other special combined characters.

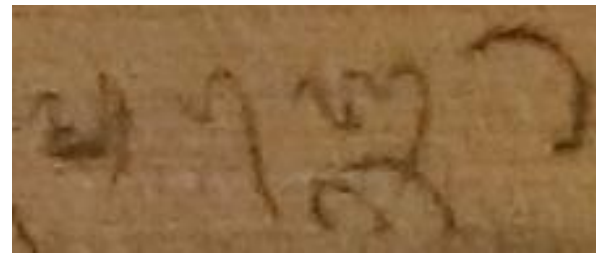

Figure 1. Balinese script on palm leaf manuscripts.

This paper is organized as follows: research method present a brief description of the DCNN architecture we proposed that was evaluated in our implementation. The palm leaf manuscript dataset used in this work and the implementation results are presented in RESULT AND DISCUSSION. Conclusions with several prospects for future work are given in CONCLUSION.

\section{RESEARCH METHOD}

\subsection{Palm leaf manuscript}

Position figures and tables at the tops and bottoms of pages, when possible. Avoid placing them in the middle of columns. Figure captions should be centered below the figures; table captions should be centered above. Avoid placing figures and tables before their first mention in the text. 
The image data that we use comes from the ICFHR competencies 2018 (http://amadi.univ-Ir.fr/ICFHR2018_Contest/index.php/challenge-3). It consists of 133

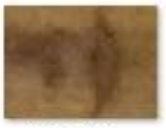

KA_65.jpg

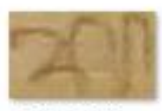

KA_66.jpg

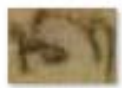

KA_78.jpg

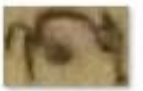

KA_90.jpg

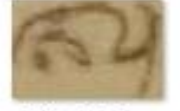

KA_67.jpg

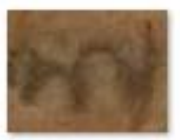

KA_79.jpg

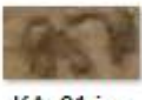

KA_91.jpg

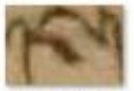

KA_103.jpg
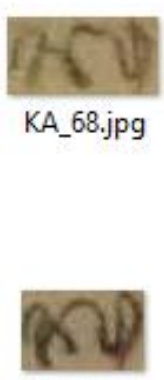

KA_80.jpg

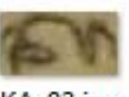

KA_92.jpg

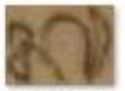

KA_104.jpg
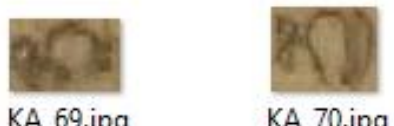

KA_70.jpg

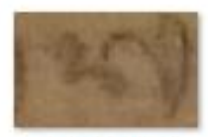

KA_81.jpg

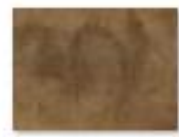

KA_82.jpg

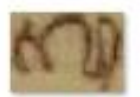

KA_93.jpg

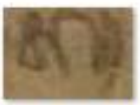

KA_94.jpg
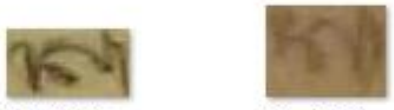

KA_105.jpg

Figure 2. Samples of character-level annotated patch images of Balinese script on palm leaf manuscripts.

character classes, with 19,383 character samples and 11,710 samples randomly selected and used as train sets. The remaining samples are used as test sets. The number of sample images for each class is different. Some classes are often found in our palm leaf manuscript collections, but some are rarely used. The thumbnail sample of these images is shown in Figure. 2.

The file received is in the form of image data collected in one folder to be used in this project so we separate each image into a folder according to the class name so that in 133 folders

\subsection{SmallerVGG}

Visual Geometry Group (VGG) is a Neural Network Architecture which won second place in ImageNet's Visual Large Scale (ILSVRC) Competition in 2014 [6]. The main contribution of this architecture shows that the depth of the number of layers of convolutional neural networks is an important component of classification accuracy. In this architecture, two convolutional layers are used sequentially with the rectified linear unit (ReLU) activation function followed by a single-pooling single layer, several layers that are fully connected with ReLU and soft-max as the last layer. The $3 \times 3$ convolutional filter with step 2 is applied to perform filtering and sampling operations simultaneously in the VGG version.

The CNN architecture we are using for this implementation is SmallerVGGNet, a simplified version of it's VGGNet. There are use 7 layers (look figure 3). We'll use width, height , depth, and classes parameters in our training script to instantiate the model with a $96 \times 96 \times$ 3 input volume. The depth specifies the number of channels in an input image, and classes is 
the number (integer) of categories/classes (not the class labels themselves). The Activation "softmax" will be utilized at the end of the network architecture. Changing this value from softmax to sigmoid will enable us to perform multi-label classification with Keras..

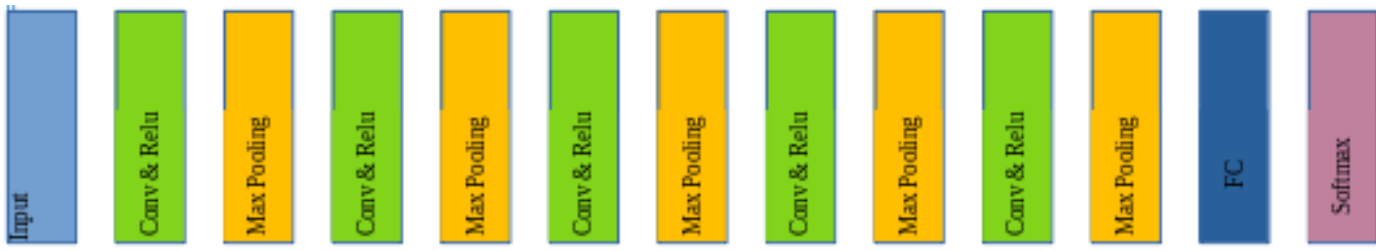

Figure 3. Aarchitecture of SmallerVGG.: Convolution (Conv) and FC for fullyconnected layers and Softmax layer at the end.

\subsection{Project Structure}

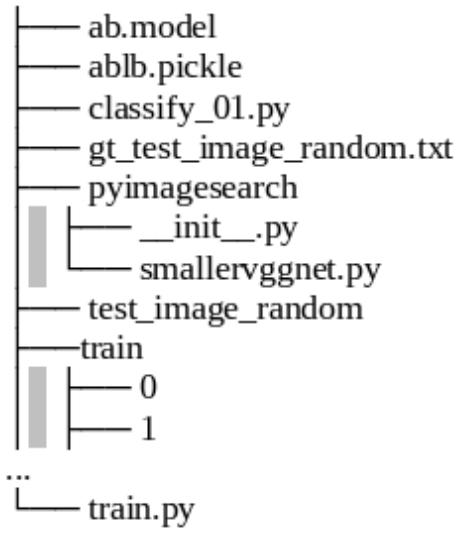

Figure 4. Project Structure Implementation SmallerVGG.

In the Project Structure, we are presented with 6 files and 3 directories. The important files we're working with (in approximate order of appearance in this article) include: 1 ) train.py : Once we've acquired the data, we'll use the train.py script to train our classifier. 2) ab.model: Our train.py script will serialize our Keras model to disk. We will use this model later in the classify.py script. 3) ablb.pickle: A scikit-learn MultiLabelBinarizer pickle file created by train.py - this file holds our class names in a convenient serialized data structure. 4)classify_01.py: In order to test our classifier. 5) gt_test_image_random.txt is our list image test with result image name

The three directories in project structure are: 1) train: This directory holds our dataset of images. Each class class has its own respective subdirectory. We do this to (1) keep our dataset organized and (2) make it easy to extract the class label name from a given image path. 2) pyimagesearch : This is our module containing our Keras neural network. Because this is a module, it contains a properly formatted _ init_.py. The other file, smallervggnet.py contains the code to assemble the neural network itself. 3)test_image_random: 7674 example images are present in this directory. We'll use classify_01.py to perform multi-label classification with Keras on each of the example images. For detail project structure we already put in https://github.com/teguhteja/cnn balinese classification. 
We use Google Collab (https://colab.research.google.com/) for running this project. Google Collab use Tesla K80 and provide GPU free without configuration that making easier to maintain this project. To test our project we have some step (look figure 5). 1) Get the image from our github. 2) Train from our image to produce model and label 3) Test model and label to get result classification. We share the result google collab

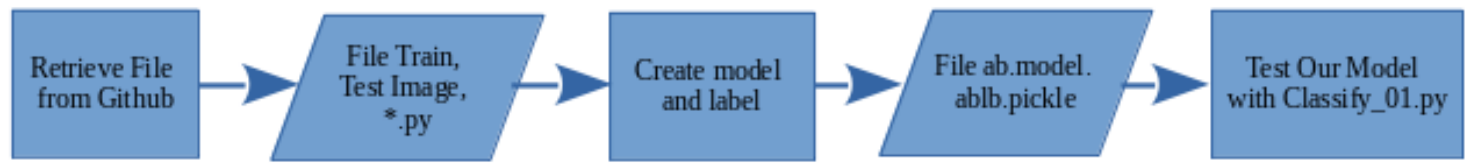

\section{RESULTS AND DISCUSSION}

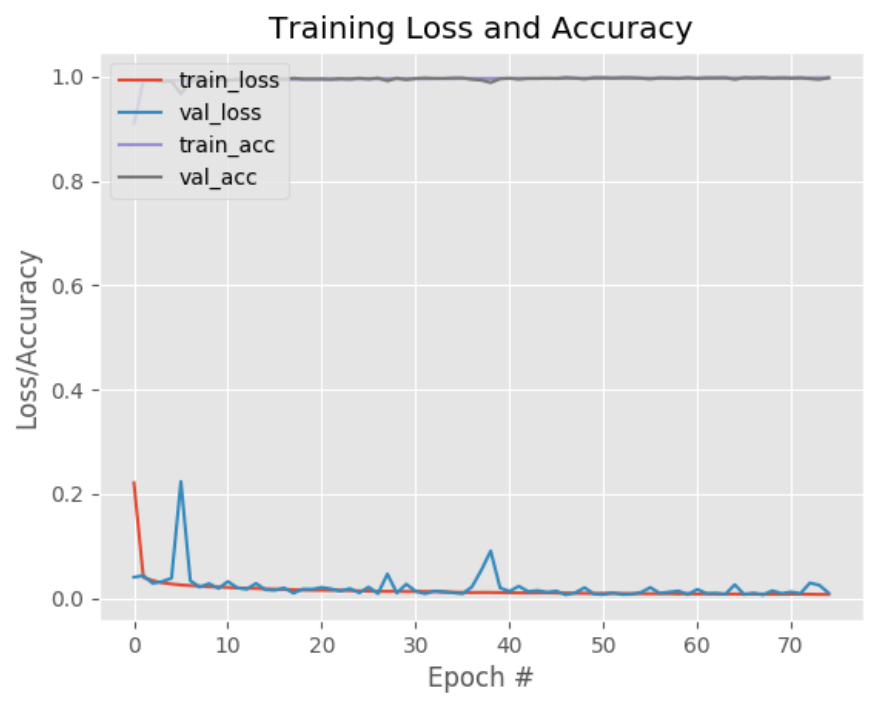

We investigated and evaluated the performance of this SmallerVGG and also compared the results of experiments with convolutional neural networks in previous studies. This dataset contains 11,730 images containing several letters and numbers. Each image is rescaled into $96 \times 96$ with some images having noise. Data is divided into $80 \%$ for training data and $20 \%$ for testing data. Using epoch 75 times we get $99.74 \%$ evaluation results for training and $99.78 \%$ for testing. To get the model and label used as validation, it takes 37 minutes in the Google Collab using GPU For the validation process using model files and labels, we classify 7674 random images with an recognition of $87.23 \%$. This result is better than the Kesiman et al of $84.30 \%$.

In our experiments, the number of training datasets for each class was not balanced, and that affected the performance of SmallerVGG. But this condition has been clearly stated and cannot be avoided as a real challenge from the development of our IHCR for Balinese 
script in palm leaf manuscripts. Some ancient characters are not often found in our palm leaf manuscript collection.

\section{CONCLUSION}

Balinese manuscripts on palm leaf manuscripts offer a new real challenge in the development of IHCR. We present our experimental study on the DCNN method for character recognition of Balinese script in palm leaf manuscripts using VGG for IHCR. We propose using SmallerVGG to increase accuracy. Our study shows that recognition rates can be significantly improved with SmallerVGG that is still slightly better than using convolutional neural networks. For our future work, we will investigate the use of other CNN architectures to achieve a better level of recognition of IHCR Balinese characters.

\section{REFERENCES}

[1] D. Cireşan, U. Meier, dan J. Schmidhuber, "Multi-column Deep Neural Networks for Image Classification," Feb 2012.

[2] C. L. Liu, F. Yin, D. H. Wang, dan Q. F. Wang, "Online and offline handwritten Chinese character recognition: Benchmarking on new databases," Pattern Recognit., vol. 46, no. 1, hal. 155-162, 2013.

[3] M. W. A. Kesiman, S. Prum, J. C. Burie, dan J. M. Ogier, "An initial study on the construction of ground truth binarized images of ancient palm leaf manuscripts," Proc. Int. Conf. Doc. Anal. Recognition, ICDAR, vol. 2015-Novem, hal. 656-660, 2015.

[4] M. W. A. Kesiman, S. Prum, J. C. Burie, dan J. M. Ogier, "Study on feature extraction methods for character recognition of Balinese script on palm leaf manuscript images," Proc. - Int. Conf. Pattern Recognit., hal. 4017-4022, 2017.

[5] K. Simonyan dan A. Zisserman, "Very Deep Convolutional Networks for Large-Scale Image Recognition," Sep 2014.

[6] M. Z. Alom, P. Sidike, M. Hasan, T. M. Taha, dan V. K. Asari, "Handwritten Bangla Character Recognition Using the State-of-the-Art Deep Convolutional Neural Networks," Comput. Intell. Neurosci., vol. 2018, hal. 1-12, 2018. 